\title{
CATEGORY-VALUED SHEAVES
}

\author{
BY JOHN W. GRAY
}

Communicated by R. Bott, March 26, 1962

This paper is a brief summary of a longer paper now in preparation. In $\$ 1$ certain notions of Freyd [1] are suitably modified so as to be immediately applicable to the theory of sheaves which is discussed in $\$ 2$.

\section{Preliminary definitions.}

Definition 1.1. A subcategory $A^{\prime}$ of a category $A$ is called left (resp., right) reflective in $A$ if there exists a functor $L: A \rightarrow A^{\prime}$ (resp., $R: A \rightarrow A^{\prime}$ ) which has the inclusion functor $A^{\prime} \rightarrow A$ as a left (resp., right) adjoint (see Kan [5]) $L$ (resp., $R$ ) is called a left (resp., right) reflection of $A$ in $A^{\prime}$.

Let $D$ be a small category (i.e., the collection of objects of $D$ is a set) and let $F(D, A)$ denote the category of (covariant) functors from $D$ to $A$ and natural transformations of such functors. Identify $A$ with the subcategory of $F(D, A)$ consisting of constant functors. A left (resp., right) reflection of $F(D, A)$ in $A$ (when it exists) will be denoted by $L r_{D}$ (resp., $R r_{D}$ ) and its values will be called left (resp., right) roots (cf. Freyd [1] and Kan [5]). Examples of left roots are direct products, difference kernels, inverse limits (inv lim), and pullbacks. Examples of right roots are direct sums, difference cokernels, direct limits (dir lim), and pushouts. All general properties of these operations are consequences of properties of roots.

Definition 1.2. If $L r_{D}: F(D, A) \rightarrow A$ (resp., $R r_{D}: F(D, A) \rightarrow A$ ) exists for every small category $D$, then $A$ is called left (resp., right) complete. A left and right complete category is called complete. A left (resp., right) complete abelian category is called left (resp., right) perfect if inverse (resp., direct) limits preserve exactness. (Cf. Axioms A B 3, A B 5 and their duals in Grothendieck [3].)

Definition 1.3. A category $A$ is called finitely generated (see Freyd [1]) if there exists a family of generators (see Grothendieck [3]) $\left\{G_{\alpha}\right\}$ such that any morphism of a $G_{\alpha}$ into the supremum of an increasingly directed family of subobjects of a given object factors through one of the subobjects.

EXAMPLES. The category of sets is finitely generated and complete. The categories of abelian groups, modules over a fixed ring, etc. are complete, right perfect, and finitely generated.

2. Sheaves. Let $X$ be a set and let $T_{X}$ be a topology on $X$ (i.e., a 
collection of open sets). Regard $T_{\boldsymbol{X}}$ as a category whose objects are the open sets $U \in T_{X}$ and with a unique morphism $U \rightarrow V$ if and only if $V \subset U$. An open covering $U$ of an open set $U \subset X$ is called a strong covering if it is closed under finite intersections.

Definition 2.1. The category $F\left(T_{X}, A\right)$ is called the category of presheaves on $X$ with values in $A$. A presheaf $F: T_{X} \rightarrow A$ is called a sheaf if given any $U \in T_{X}$ and any strong covering $\left\{U_{\alpha}\right\}$ of $U$, we have $F(U)=$ inv $\lim F\left(U_{\alpha}\right) . S(X \cdot A)$ will denote the full subcategory of $F\left(T_{X}, A\right)$ determined by all sheaves, and will be called the category of sheaves on $X$ with values in $A$.

If $f: X \rightarrow Y$ is a continuous map, then $f$ induces a functor $\bar{f}: T_{Y} \rightarrow T_{X}$ by the rule $\bar{f}(V)=f^{-1}(V)$ for $V \in T_{Y}$. Composition on the "left" with $\bar{f}$ determines a functor $f_{*}: F\left(T_{X}, A\right) \rightarrow F\left(T_{Y}, A\right)$ (i.e., if $F: T_{X} \rightarrow A$ then $f_{*} F=F \circ f$; diagrammatically, $T_{Y} \rightarrow T_{X} \rightarrow A$; hence "left" above). Since composition on the left preserves left roots, $f_{*}$ takes sheaves on $X$ into sheaves on $Y$. Nevertheless, we regard $f_{*}$ as a functor $f_{*}: S(X, A)$ $\rightarrow F\left(T_{Y}, A\right)$. Our main aim is to construct a left adjoint $f^{*}$ to the functor $f_{*}$ (see Grothendieck [4]).

Let $A$ be right complete and let $F: T_{X} \rightarrow A$ be a presheaf. For each $x \in X$, let $F_{x}=\operatorname{dir} \lim _{x \in U} F(U)$ and let $\rho_{x, U}: F(U) \rightarrow F_{x}$ be the canonical morphism. By definition of left roots there is a unique $\rho_{U}: F(U)$ $\rightarrow \prod_{x \in U} F_{x}$ such that $p_{x} \circ \rho_{U}=\rho_{x, U}$, where $p_{x}: \prod_{x \in U} F_{x} \rightarrow F_{x}$ is the projection onto the $x$ th factor. Let $P_{X} F$ be the presheaf such that $P_{X} F(U)=\prod_{x \in U} F_{x}$. It is easily checked that $P_{X} F$ is a sheaf and that $\rho_{F}: F \rightarrow P_{X} F$ (where the components of $\rho_{F}$ are the morphisms $\rho_{U}$ above) is a morphism of presheaves.

Lемма 2.2. Let $A$ be complete and finitely generated. If $F$ is a sheaf then $\rho_{F}: F \rightarrow P_{X} F$ is a monomorphism.

MaIn Theorem. Let $A$ be complete and finitely generated.

(i) $f_{*}: S(X, A) \rightarrow F\left(T_{Y}, A\right)$ has a left adjoint $f^{*}: F\left(T_{Y}, A\right) \rightarrow S(X, A)$. For any $F \in F\left(T_{Y}, A\right)$, we have $\left(f^{*} F\right)_{x}=F_{f(x)}$.

(ii) If, in addition, $A$ is abelian and right perfect, then $S(X, A)$ is abelian, complete, right perfect, and finitely generated. $f^{*}$ is exact, and furthermore, a sequence $0 \rightarrow F^{\prime} \rightarrow F \rightarrow F^{\prime \prime} \rightarrow 0$ in $S(X, A)$ is exact if and only if $0 \rightarrow F_{x}^{\prime} \rightarrow F_{x} \rightarrow F_{x}^{\prime \prime} \rightarrow 0$ is exact in $A$ for every $x \in X$.

REMARKs. (i) If $f$ is the identity map $i: X \rightarrow X$, then $i_{*}$ is just the inclusion $S(X, A) \rightarrow F\left(T_{X}, A\right)$. Statement (i) then asserts that the category of sheaves is a right reflective subcategory of the category of presheaves. $i^{*} F$ is the sheaf associated with the presheaf $F$. The proof in this case is extraordinarily simple. Essentially, $i^{*} F$ is the 
intersection of all subsheaves of $P_{x} F$ through which $\rho_{F}: F \rightarrow P_{X} F$ factors.

(ii) The rest of sheaf theory now follows naturally. For example, to find the sheaf agreeing with a given sheaf $F$ on a locally closed subspace $A \subset X$ and zero on $X-A$; consider two cases: (a) $A$ is closed; then $j_{*} j^{*} F$ has the desired properties (where $j: A \rightarrow X$ is the inclusion). Furthermore, $F \rightarrow j_{*} j^{*} F$ is an epimorphism. (b) $A$ open. Let $k:(X-A)$ $\rightarrow X$ be the inclusion. Then the kernel of the epimorphism $F \rightarrow k_{*} k^{*} F$ is the desired sheaf. (See Godement [2].)

Duality theorems are handled by considering cosheaves; i.e., sheaves with values in the dual category to a given category. Cech cohomology is definable and, with suitable modifications, occurs in the usual spectral sequences. To obtain the full strength of the Cech theory one must add to $A$ the hypothesis that direct products are exact.

\section{REFERENCES}

1. Peter J. Freyd, Functor theory, Thesis, Princeton University, 1960.

2. R. Godement, Topologie algébrique et theorie des faisceaux, Hermann, Paris, 1958.

3. A. Grothendieck, Sur quelques points d'algèbre homologique, Tôhoku Math. J. 9 (1957), 119-221.

4. - Éléments de géométrie algébrique I, Inst. Hautes Études Sci. Publ. Math. No. 4 (1960).

5. D. Kan, Adjoint functors, Trans. Amer. Math. Soc. 87 (1958), 294-329.

Columbia University 